RM3-TH/12-5

\title{
On the NLO QCD corrections to the production of the heaviest neutral Higgs scalar in the MSSM
}

\author{
G. Degrassi ${ }^{a}$, S. Di Vita ${ }^{a}$ and P. Slavich ${ }^{b}$ \\ ${ }^{a}$ Dipartimento di Fisica, Università di Roma Tre and INFN, Sezione di Roma Tre \\ Via della Vasca Navale 84, I-00146 Rome, Italy \\ ${ }^{b}$ LPTHE, 4, Place Jussieu, F-75252 Paris, France
}

\begin{abstract}
We present a calculation of the two-loop top-stop-gluino contributions to Higgs production via gluon fusion in the MSSM. By means of an asymptotic expansion in the heavy particle masses, we obtain explicit and compact analytic formulae that are valid when the Higgs and the top quark are lighter than stops and gluino, without assuming a specific hierarchy between the Higgs mass and the top mass. Being applicable to the heaviest Higgs scalar in a significant region of the MSSM parameter space, our results complement earlier ones obtained with a Taylor expansion in the Higgs mass, and can be easily implemented in computer codes to provide an efficient and accurate determination of the Higgs production cross section.
\end{abstract}

e-mail:

degrassi@fis.uniroma3.it

divita@is.uniroma3.it

slavich@lpthe.jussieu.fr 


\section{Introduction}

With the coming into operation of the Large Hadron Collider (LHC), a new era has begun in the search for the Higgs boson(s). This search requires an accurate control of all the Higgs production and decay mechanisms, including the effects due to radiative corrections [1]. At the LHC the main production mechanism for the Standard Model (SM) Higgs boson, $H_{\mathrm{SM}}$, is the loop-induced gluon fusion mechanism [2], $g g \rightarrow H_{\mathrm{SM}}$, where the coupling of the gluons to the Higgs is mediated by loops of colored fermions, primarily the top quark. The knowledge of this process in the SM includes the full next-to-leading order (NLO) QCD corrections [3, 4, 5, 6, 7, 8]; the next-to-next-to-leading order (NNLO) QCD corrections [9] including finite top mass effects [10]; soft-gluon resummation effects [11; the first-order electroweak (EW) corrections [12, 13, 14]; estimates of the next-to-next-to-nextto-leading order (NNNLO) QCD corrections [15] and of the mixed QCD-EW corrections [16].

The Higgs sector of the Minimal Supersymmetric Standard Model (MSSM) consists of two $S U(2)$ doublets, $H_{1}$ and $H_{2}$, whose relative contribution to electroweak symmetry breaking is determined by the ratio of vacuum expectation values of their neutral components, $\tan \beta \equiv v_{2} / v_{1}$. The spectrum of physical Higgs bosons is richer than in the SM, consisting of two neutral CP-even bosons, $h$ and $H$, one

neutral CP-odd boson, $A$, and two charged bosons, $H^{ \pm}$. The couplings of the MSSM Higgs bosons to matter fermions differ from those of the SM Higgs, and they can be considerably enhanced (or suppressed) depending on $\tan \beta$. As in the SM, gluon fusion is one of the most important production mechanisms for the neutral Higgs bosons, whose couplings to the gluons are mediated by top and bottom quarks and their supersymmetric partners, the stop and sbottom squarks.

In the MSSM, the cross section for Higgs boson production in gluon fusion is currently known at the NLO. The contributions arising from diagrams with quarks and gluons, with full dependence on the Higgs and quark masses, can be obtained from the corresponding SM results [4, 5, 6, 7, 8] with an appropriate rescaling of the Higgs-quark couplings. The contributions arising from diagrams with squarks and gluons were first computed under the approximation of vanishing Higgs mass in ref. [17], and the full Higgs-mass dependence was included in later calculations [6, 7, 8, 18]. The contributions of two-loop diagrams involving top, stop and gluino to both scalar and pseudoscalar Higgs production were computed in the vanishing-Higgs-mass limit (VHML) in refs. [19, 20], whose results were later confirmed and cast in a compact analytic form in refs. [21, 22]. Finally, first results for the NNLO contributions in the limit of vanishing Higgs mass and degenerate stop and gluino masses were presented in ref. [23].

The VHML can provide reasonably accurate results as long as the Higgs mass is well below the threshold for creation of the massive particles running in the loops. For the production of the lightest scalar Higgs, this condition does apply to the two-loop diagrams involving top, stop and gluino, but it obviously does not apply to the corresponding diagrams involving the bottom quark, whose contribution can be relevant for large values of $\tan \beta$. In turn, the masses of the heaviest scalar $H$ and of the pseudoscalar $A$ might very well approach (or exceed) the threshold for creation of top quarks or even of squarks. Unfortunately, retaining the full dependence on the Higgs mass in the quark- 
squark-gluino contributions has proved a rather daunting task. A calculation based on a combination of analytic and numerical methods was presented in ref. 24] (see also ref. [25]), but neither explicit analytic results nor a public computer code have been made available so far.

However, results from the first year of supersymmetry (SUSY) searches at the LHC (see, e.g., ref. [26]) set preliminary lower bounds on the squark and gluino masses of the order of the TeV, albeit for specific models of SUSY breaking. This suggests that - if the MSSM is actually realized in nature - there might be wide regions of its parameter space in which all three of the neutral Higgs bosons are somewhat lighter than the squarks and the gluino. Approximate analytic results for the quark-squark-gluino contributions can be derived in this case. In particular, ref. [27] presented an approximate evaluation of the bottom-sbottom-gluino contributions to scalar production, based on an asymptotic expansion in the large supersymmetric masses that is valid up to and including terms of $\mathcal{O}\left(m_{b}^{2} / m_{\phi}^{2}\right), \mathcal{O}\left(m_{b} / M\right)$ and $\mathcal{O}\left(m_{z}^{2} / M^{2}\right)$, where $m_{\phi}$ denotes a Higgs boson mass and $M$ denotes a generic superparticle mass. An independent calculation of the quark-squark-gluino contributions to scalar production, restricted to the limit of zero squark mixing and degenerate superparticle masses, was also presented in ref. [28], confirming the results of ref. [27] for the bottom contributions. More recently, ref. [22] presented an evaluation of the quark-squark-gluino contributions to pseudoscalar production that is also based on an asymptotic expansion in the large supersymmetric masses, but does not assume any hierarchy between the pseudoscalar mass and the quark mass, thus covering both the top-stop-gluino and bottom-sbottom-gluino cases.

Exploiting the asymptotic-expansion techniques developed in refs. [27] and [22], we provide in this paper an evaluation of the two-loop top-stop-gluino contributions to Higgs-scalar production valid when the Higgs and the top quark are lighter than stops and gluino, without assuming a specific hierarchy between the Higgs mass and the top mass. In particular, we provide explicit and compact analytic formulae which include terms up to $\mathcal{O}\left(m_{\phi}^{2} / M^{2}\right), \mathcal{O}\left(m_{t}^{2} / M^{2}\right)$ and $\mathcal{O}\left(m_{z}^{2} / M^{2}\right)$. The results presented in this paper complement the earlier ones of ref. [21], which, being obtained via a Taylor expansion in the Higgs mass, are not accurate for a Higgs mass comparable to (or greater than) the top mass, as might well be the case for the heaviest Higgs scalar of the MSSM. Our formulae can be easily implemented in computer codes 1 , allowing for an efficient and accurate determination of the Higgs-boson production cross section in the MSSM.

The paper is organized as follows: in section 2 we summarize general results on the form factors for Higgs boson production via gluon fusion in the MSSM. Section 3 contains our explicit results for the contributions arising from two-loop top-stop-gluino diagrams, as well as a discussion of the renormalization conditions for the parameters in the top/stop sector. In section 4 we compare numerically the results of our asymptotic expansion in the heavy masses with the results of a Taylor expansion in the Higgs mass, up to and including terms of $\mathcal{O}\left(m_{\phi}^{2} / m_{t}^{2}\right)$ and $\mathcal{O}\left(m_{\phi}^{2} / M^{2}\right)$, discussing the regions of applicability of the two different expansions and the effect of different renormalization conditions. Finally, in the last section we present our conclusions.

\footnotetext{
${ }^{1}$ An implementation of the MSSM gluon-fusion cross section in the POWHEG framework was presented in ref. [29].
} 


\section{Higgs boson production via gluon fusion in the MSSM}

In this section we recall for completeness some general results on Higgs boson production via gluon fusion in the MSSM. The leading-order (LO) partonic cross section for the $g g \rightarrow \phi$ process (with $\phi=h, H)$ reads

$$
\sigma^{(0)}=\frac{G_{\mu} \alpha_{s}^{2}\left(\mu_{R}\right)}{128 \sqrt{2} \pi}\left|\mathcal{H}_{\phi}^{1 \ell}\right|^{2}
$$

where $G_{\mu}$ is the muon decay constant, $\alpha_{s}\left(\mu_{R}\right)$ is the strong gauge coupling expressed in the $\overline{\mathrm{MS}}$ renormalization scheme at the scale $\mu_{R}$, and $\mathcal{H}_{\phi}$ is the form factor for the coupling of the CP-even Higgs boson $\phi$ with two gluons, which we decompose in one- and two-loop parts as

$$
\mathcal{H}_{\phi}=\mathcal{H}_{\phi}^{1 \ell}+\frac{\alpha_{s}}{\pi} \mathcal{H}_{\phi}^{2 \ell}+\mathcal{O}\left(\alpha_{s}^{2}\right)
$$

The form factors for the lightest and heaviest Higgs mass eigenstates can be decomposed as

$$
\mathcal{H}_{h}=T_{F}\left(-\sin \alpha \mathcal{H}_{1}+\cos \alpha \mathcal{H}_{2}\right), \quad \mathcal{H}_{H}=T_{F}\left(\cos \alpha \mathcal{H}_{1}+\sin \alpha \mathcal{H}_{2}\right)
$$

where $T_{F}=1 / 2$ is a color factor, $\alpha$ is the mixing angle in the CP-even Higgs sector of the MSSM and $\mathcal{H}_{i}(i=1,2)$ are the form factors for the coupling of the neutral, CP-even component of the Higgs doublet $H_{i}$ with two gluons. Focusing on the contributions involving the third-generation quarks and squarks, and exploiting the structure of the Higgs-quark-quark and Higgs-squark-squark couplings, we can write to all orders in the strong interactions 21]

$$
\begin{aligned}
& \mathcal{H}_{1}=\lambda_{t}\left[m_{t} \mu s_{2 \theta_{t}} F_{t}+m_{Z}^{2} s_{2 \beta} D_{t}\right]+\lambda_{b}\left[m_{b} A_{b} s_{2 \theta_{b}} F_{b}+2 m_{b}^{2} G_{b}+2 m_{Z}^{2} c_{\beta}^{2} D_{b}\right], \\
& \mathcal{H}_{2}=\lambda_{b}\left[m_{b} \mu s_{2 \theta_{b}} F_{b}-m_{Z}^{2} s_{2 \beta} D_{b}\right]+\lambda_{t}\left[m_{t} A_{t} s_{2 \theta_{t}} F_{t}+2 m_{t}^{2} G_{t}-2 m_{Z}^{2} s_{\beta}^{2} D_{t}\right] .
\end{aligned}
$$

In the equations above $\lambda_{t}=1 / \sin \beta$ and $\lambda_{b}=1 / \cos \beta$. Also, $\mu$ is the higgsino mass parameter in the MSSM superpotential, $A_{q}$ (for $q=t, b$ ) are the soft SUSY-breaking Higgs-squark-squark couplings and $\theta_{q}$ are the left-right squark mixing angles (here and thereafter we use the notation $s_{\varphi} \equiv \sin \varphi, c_{\varphi} \equiv$ $\cos \varphi$ for a generic angle $\varphi$ ). The functions $F_{q}$ and $G_{q}$ appearing in eqs. (4) and (5I) denote the contributions controlled by the third-generation Yukawa couplings, while $D_{q}$ denotes the contribution controlled by the electroweak, D-term-induced Higgs-squark-squark couplings. The latter can be decomposed as

$$
D_{q}=\frac{I_{3 q}}{2} \widetilde{G}_{q}+c_{2 \theta_{\tilde{q}}}\left(\frac{I_{3 q}}{2}-Q_{q} s_{\theta_{W}}^{2}\right) \widetilde{F}_{q},
$$

where $I_{3 q}$ denotes the third component of the electroweak isospin of the quark $q, Q_{q}$ is the electric charge and $\theta_{W}$ is the Weinberg angle.

The form factors $\mathcal{H}_{i}$ can in turn be decomposed in one- and two-loop parts as in eq. (2). The one-loop parts, $\mathcal{H}_{i}^{1 \ell}$, contain contributions from diagrams involving quarks $(q)$ or squarks $\left(\tilde{q}_{i}\right)$. The functions entering $\mathcal{H}_{i}^{1 \ell}$ are 


$$
\begin{aligned}
F_{q}^{1 \ell}=\widetilde{F}_{q}^{1 \ell} & =\frac{1}{2}\left[\frac{1}{m_{\tilde{q}_{1}}^{2}} \mathcal{G}_{0}^{1 \ell}\left(\tau_{\tilde{q}_{1}}\right)-\frac{1}{m_{\tilde{q}_{2}}^{2}} \mathcal{G}_{0}^{1 \ell}\left(\tau_{\tilde{q}_{2}}\right)\right] \\
G_{q}^{1 \ell} & =\frac{1}{2}\left[\frac{1}{m_{\tilde{q}_{1}}^{2}} \mathcal{G}_{0}^{1 \ell}\left(\tau_{\tilde{q}_{1}}\right)+\frac{1}{m_{\tilde{q}_{2}}^{2}} \mathcal{G}_{0}^{1 \ell}\left(\tau_{\tilde{q}_{2}}\right)+\frac{1}{m_{q}^{2}} \mathcal{G}_{1 / 2}^{1 \ell}\left(\tau_{q}\right)\right], \\
\widetilde{G}_{q}^{1 \ell} & =\frac{1}{2}\left[\frac{1}{m_{\tilde{q}_{1}}^{2}} \mathcal{G}_{0}^{1 \ell}\left(\tau_{\tilde{q}_{1}}\right)+\frac{1}{m_{\tilde{q}_{2}}^{2}} \mathcal{G}_{0}^{1 \ell}\left(\tau_{\tilde{q}_{2}}\right)\right]
\end{aligned}
$$

where $\tau_{k} \equiv 4 m_{k}^{2} / m_{h}^{2}$, and the functions $\mathcal{G}_{0}^{1 \ell}$ and $\mathcal{G}_{1 / 2}^{1 \ell}$ read

$$
\begin{aligned}
\mathcal{G}_{0}^{1 \ell}(\tau) & =\tau\left[1+\frac{\tau}{4} \ln ^{2}\left(\frac{\sqrt{1-\tau}-1}{\sqrt{1-\tau}+1}\right)\right], \\
\mathcal{G}_{1 / 2}^{1 \ell}(\tau) & =-2 \tau\left[1-\frac{1-\tau}{4} \ln ^{2}\left(\frac{\sqrt{1-\tau}-1}{\sqrt{1-\tau}+1}\right)\right] .
\end{aligned}
$$

The analytic continuations are obtained with the replacement $m_{h}^{2} \rightarrow m_{h}^{2}+i \epsilon$. We remark that in the limit in which the Higgs boson mass is much smaller than the mass of the particle running in the loop, i.e. $\tau \gg 1$, the functions $\mathcal{G}_{0}^{1 \ell}$ and $\mathcal{G}_{1 / 2}^{1 \ell}$ behave as

$$
\mathcal{G}_{0}^{1 \ell} \rightarrow-\frac{1}{3}-\frac{8}{45 \tau}+\mathcal{O}\left(\tau^{-2}\right), \quad \mathcal{G}_{1 / 2}^{1 \ell} \rightarrow-\frac{4}{3}-\frac{14}{45 \tau}+\mathcal{O}\left(\tau^{-2}\right) .
$$

The two-loop parts of the form factors, $\mathcal{H}_{i}^{2 \ell}$, contain contributions from diagrams involving quarks, squarks, gluons and gluinos. We point the reader to, e.g., section 2 of ref. [21] for explicit formulae showing how $\mathcal{H}_{i}^{2 \ell}$ (or, equivalently, $\mathcal{H}_{\phi}^{2 \ell}$ ) enter the total NLO cross section for Higgs boson production in hadronic collisions. In the next section we present our new evaluation of the top/stop contributions to $\mathcal{H}_{i}^{2 \ell}$, based on an asymptotic expansion in the stop and gluino masses.

\section{Two-loop contributions to the Higgs-production form factors}

In the case of the lightest Higgs boson $h$, the top/stop contributions to the two-loop form factor $\mathcal{H}_{h}^{2 \ell}$ are well under control. Typically, the mass ratios between the lightest Higgs and the particles running in the loops allow for the evaluation of the relevant diagrams via a Taylor expansion in the Higgs mass, with the zero-order term in the series - for which ref. [21] provides explicit analytic formulae - already a good approximation to the full result. In the case of the heaviest Higgs boson $H$, on the other hand, the assumption that it is much lighter than the particles running in the loops is valid only in a limited portion of the MSSM parameter space. In particular, $m_{H}$ might very well sit around or above the threshold for the creation of a real top-quark pair in the loops, in which case - as found in ref. [22] for the pseudoscalar - a Taylor expansion in $m_{H}^{2}$ would certainly fail to approximate the correct result for the Higgs-production form factor. To address this possibility, we present in this section explicit analytic results for the two-loop top/stop contributions to the form factors $\mathcal{H}_{i}^{2 \ell}$ that include terms up 

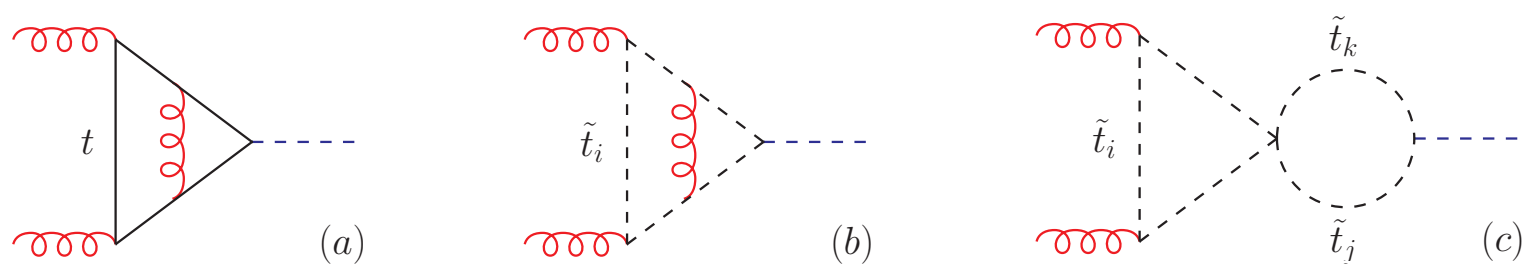

Figure 1: Examples of two-loop diagrams for $g g \rightarrow \phi$ that do not involve gluinos.
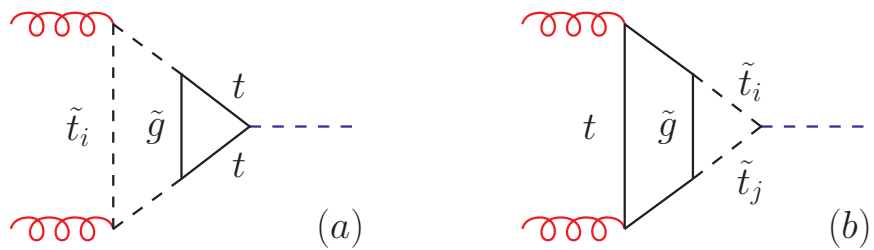

Figure 2: Examples of two-loop diagrams for $g g \rightarrow \phi$ involving gluinos.

to $\mathcal{O}\left(m_{\phi}^{2} / M^{2}\right), \mathcal{O}\left(m_{z}^{2} / M^{2}\right)$ and $\mathcal{O}\left(m_{t}^{2} / M^{2}\right)$, without assuming a specific hierarchy between the Higgs mass and the top mass.

The top/stop contributions to $\mathcal{H}_{i}^{2 \ell}$ come from two-loop diagrams such as the ones depicted in figs. 1 and 2. In analogy to what was done in refs. [21, 27], we can decompose the functions $F_{t}^{2 \ell}, G_{t}^{2 \ell}, \widetilde{F}_{t}^{2 \ell}$ and $\widetilde{G}_{t}^{2 \ell}$ entering the two-loop parts of eqs. (41) and (5) as

$$
\begin{aligned}
F_{t}^{2 \ell} & =Y_{\tilde{t}_{1}}-Y_{\tilde{t}_{2}}-\frac{4 c_{2 \theta_{t}}^{2}}{m_{\tilde{t}_{1}}^{2}-m_{\tilde{t}_{2}}^{2}} Y_{c_{2 \theta_{t}}^{2}}, \\
G_{t}^{2 \ell} & =Y_{\tilde{t}_{1}}+Y_{\tilde{t}_{2}}+Y_{t}, \\
\widetilde{F}_{t}^{2 \ell} & =Y_{\tilde{t}_{1}}-Y_{\tilde{t}_{2}}+\frac{4 s_{2 \theta_{t}}^{2}}{m_{\tilde{t}_{1}}^{2}-m_{\tilde{t}_{2}}^{2}} Y_{c_{2 \theta_{t}}^{2}}, \\
\widetilde{G}_{t}^{2 \ell} & =Y_{\tilde{t}_{1}}+Y_{\tilde{t}_{2}} .
\end{aligned}
$$

The various terms in eqs. (13) $-(16)$ can be split in the contributions coming from diagrams with

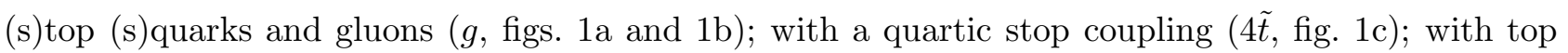
quarks, stop squarks and gluinos ( $\tilde{g}$, figs. 2a and 2b):

$$
Y_{x}=Y_{x}^{g}+Y_{x}^{4 \tilde{t}}+Y_{x}^{\tilde{g}} \quad\left(x=t, \tilde{t}_{1}, \tilde{t}_{2}, c_{2 \theta_{t}}^{2}\right) .
$$

Furthermore, we remark that the term $Y_{t}$ entering eq. (14) contains only contributions from diagrams with a Higgs-top coupling, figs. 11 and 2 a, therefore $Y_{t}^{4 \tilde{t}}=0$. On the other hand, the terms $Y_{\tilde{t}_{1}}, Y_{\tilde{t}_{2}}$ and $Y_{c_{2 \theta_{t}}^{2}}$ in eqs. (13)-(16) contain only contributions from diagrams with a Higgs-stop coupling, figs. 1 $\mathrm{b}$, 11;, and 2]b. 


\subsection{Top-gluon, stop-gluon and four-stop contributions}

The top-gluon, stop-gluon and four-stop contributions to the terms $Y_{x}$ in eq. (17) can be extracted from the existing literature, and we collect them in this section for completeness. We assume that the parameters entering the one-loop parts of the form factors $\mathcal{H}_{i}$ in eqs. (44) and (5) are expressed in the $\overline{\mathrm{DR}}$ renormalization scheme at the scale $Q$.

The contribution to the term $Y_{t}$ arising from two-loop diagrams with top quarks and gluons (fig. 1a) must be computed for arbitrary values of $\tau_{t} \equiv 4 m_{t}^{2} / m_{\phi}^{2}$. It reads:

$$
2 m_{t}^{2} Y_{t}^{g}=C_{F}\left[\mathcal{F}_{1 / 2}^{(2 \ell, a)}\left(x_{t}\right)+\mathcal{F}_{1 / 2}^{(2 \ell, b)}\left(x_{t}\right)\left(\ln \frac{m_{t}^{2}}{Q^{2}}-\frac{1}{3}\right)\right]+C_{A} \mathcal{G}_{1 / 2}^{\left(2 \ell, C_{A}\right)}\left(x_{t}\right)
$$

where $C_{F}=4 / 3$ and $C_{A}=3$ are color factors, and exact expressions for $\mathcal{F}_{1 / 2}^{(2 \ell, a)}, \mathcal{F}_{1 / 2}^{(2 \ell, b)}$ and $\mathcal{G}_{1 / 2}^{\left(2 \ell, C_{A}\right)}$ as functions of $x_{t} \equiv\left(\sqrt{1-\tau_{t}}-1\right) /\left(\sqrt{1-\tau_{t}}+1\right)$ are given in eqs. (2.12), (2.13) and (3.8) of ref. [7], respectively.

The contributions to the terms $Y_{\tilde{t}_{1}}, Y_{\tilde{t}_{2}}$ and $Y_{c_{2 \theta_{t}}^{2}}$ arising from two-loop diagrams with stop squarks and gluons (fig. 1b) and from diagrams with a quartic stop coupling (fig. 1c) can, to the accuracy required by our expansion, be computed in the limit of vanishing $m_{\phi}$. They read [21]

$$
\begin{aligned}
Y_{\tilde{t}_{1}}^{g} & =-\frac{1}{2 m_{\tilde{t}_{1}}^{2}}\left(\frac{3 C_{F}}{4}+\frac{C_{A}}{6}\right), \\
Y_{\tilde{t}_{1}}^{4 \tilde{t}} & =-\frac{C_{F}}{24}\left[\frac{c_{2 \theta_{t}}^{2} m_{\tilde{t}_{1}}^{2}+s_{2 \theta_{t}}^{2} m_{\tilde{t}_{2}}^{2}}{m_{\tilde{t}_{1}}^{4}}+\frac{s_{2 \theta_{t}}^{2}}{m_{\tilde{t}_{1}}^{4} m_{\tilde{t}_{2}}^{2}}\left(m_{\tilde{t}_{1}}^{4} \ln \frac{m_{\tilde{t}_{1}}^{2}}{Q^{2}}-m_{\tilde{t}_{2}}^{4} \ln \frac{m_{\tilde{t}_{2}}^{2}}{Q^{2}}\right)\right], \\
Y_{c_{2 \theta_{t}}}^{4 \tilde{t}} & =-\frac{C_{F}}{24}\left[\frac{\left(m_{\tilde{t}_{1}}^{2}-m_{\tilde{t}_{2}}^{2}\right)^{2}}{m_{\tilde{t}_{1}}^{2} m_{\tilde{t}_{2}}^{2}}-\frac{m_{\tilde{t}_{1}}^{2}-m_{\tilde{t}_{2}}^{2}}{m_{\tilde{t}_{2}}^{2}} \ln \frac{m_{\tilde{t}_{1}}^{2}}{Q^{2}}-\frac{m_{\tilde{t}_{2}}^{2}-m_{\tilde{t}_{1}}^{2}}{m_{\tilde{t}_{1}}^{2}} \ln \frac{m_{\tilde{t}_{2}}^{2}}{Q^{2}}\right] .
\end{aligned}
$$

The term $Y_{c_{2 \theta_{t}}^{2}}^{g}$ is zero, while the terms $Y_{\tilde{t}_{2}}^{g}$ and $Y_{\tilde{t}_{2}}^{4 \tilde{t}}$ can be obtained by performing the substitutions $\tilde{t}_{1} \leftrightarrow \tilde{t}_{2}$ in eqs. (19) and (20), respectively.

\section{$3.2 \quad$ Top-stop-gluino contributions}

In this section we present our original results for the asymptotic expansion of the top-stop-gluino contributions in the stop and gluino masses. We retain in our formulae only terms that contribute to the form factors $\mathcal{H}_{i}$ up to $\mathcal{O}\left(m_{t}^{2} / M^{2}\right), \mathcal{O}\left(m_{\phi}^{2} / M^{2}\right)$ or $\mathcal{O}\left(m_{z}^{2} / M^{2}\right)$, where $M$ denotes a generic superparticle mass. Again, we assume that the one-loop parts of $\mathcal{H}_{i}$ in eqs. (4) and (5) are expressed in terms of $\overline{\mathrm{DR}}$-renormalized parameters evaluated at the scale $Q$. The top-stop-gluino contributions to the term $Y_{t}$, arising from diagrams with a Higgs-top coupling (fig. $2 \mathrm{a}$ ), read

$$
\begin{aligned}
2 m_{t}^{2} Y_{t}^{\tilde{g}}= & \frac{4}{3} \mathcal{F}_{1 / 2}^{(2 \ell, b)}\left(\tau_{t}\right){\frac{\delta m_{t}}{m_{t}}}^{S U S Y}-\frac{C_{F}}{4} \mathcal{G}_{1 / 2}^{1 \ell}\left(\tau_{t}\right) \frac{m_{\tilde{g}}}{m_{t}} s_{2 \theta_{t}}\left(\frac{x_{1}}{1-x_{1}} \ln x_{1}-\frac{x_{2}}{1-x_{2}} \ln x_{2}\right) \\
& +s_{2 \theta_{t}} \frac{m_{t}}{m_{\tilde{g}}} \mathcal{R}_{1}+\frac{m_{t}^{2}}{m_{\tilde{g}}^{2}} \mathcal{R}_{2},
\end{aligned}
$$


where $x_{i}=m_{\tilde{t}_{i}}^{2} / m_{\tilde{g}}^{2}(i=1,2)$, and $\delta m_{t}^{S U S Y}$ denotes the SUSY contribution to the top self-energy, in units of $\alpha_{s} / \pi$, expanded in powers of $m_{t}$ up to terms of $\mathcal{O}\left(m_{t}^{3}\right)$

$$
\begin{aligned}
\delta m_{t}^{S U S Y} & =-\frac{C_{F}}{4} m_{t}\left[s_{2 \theta_{t}} \frac{m_{\tilde{g}}}{m_{t}} \frac{x_{1}}{1-x_{1}} \ln x_{1}+\frac{1}{2} \ln \frac{m_{\tilde{g}}^{2}}{Q^{2}}+\frac{x_{1}-3}{4\left(1-x_{1}\right)}+\frac{x_{1}\left(x_{1}-2\right)}{2\left(1-x_{1}\right)^{2}} \ln x_{1}\right. \\
& \left.+\frac{s_{2 \theta_{t}} m_{t}}{2 m_{\tilde{g}}\left(1-x_{1}\right)^{3}}\left(1-x_{1}^{2}+2 x_{1} \ln x_{1}\right)+\frac{m_{t}^{2}}{6 m_{\tilde{g}}^{2}\left(1-x_{1}\right)^{3}}\left(x_{1}^{2}-5 x_{1}-2-\frac{6 x_{1}}{1-x_{1}} \ln x_{1}\right)\right] \\
& +\left(x_{1} \longrightarrow x_{2}, \quad s_{2 \theta_{t}} \longrightarrow-s_{2 \theta_{t}}\right) .
\end{aligned}
$$

The terms $\mathcal{R}_{1}$ and $\mathcal{R}_{2}$ in eq. (22) collect contributions suppressed by $m_{t} / M$ and $m_{t}^{2} / M^{2}$, respectively:

$$
\begin{aligned}
& \mathcal{R}_{1}=\frac{C_{A}}{6\left(1-x_{1}\right)^{2}}\left[3\left(1-x_{1}+x_{1} \ln x_{1}\right)\left(\ln \frac{m_{t}^{2}}{m_{\tilde{g}}^{2}}-\mathcal{B}\left(\tau_{t}\right)-\frac{1}{2} \mathcal{K}^{1 \ell}\left(\tau_{t}\right)+2\right)\right. \\
& \left.+6 x_{1} \operatorname{Li}_{2}\left(1-x_{1}\right)+2 x_{1}+2 x_{1}\left(1+x_{1}\right) \ln x_{1}-2\right] \\
& -\frac{C_{F}}{6 x_{1}\left(1-x_{1}\right)^{3}}\left[3\left(x_{1}-x_{1}^{3}+2 x_{1}^{2} \ln x_{1}\right)\left(\ln \frac{m_{t}^{2}}{m_{\tilde{g}}^{2}}-\mathcal{B}\left(\tau_{t}\right)-\frac{1}{4} \mathcal{G}_{1 / 2}^{1 \ell}\left(\tau_{t}\right)-\frac{1}{2} \mathcal{K}^{1 \ell}\left(\tau_{t}\right)+2\right)\right. \\
& \left.+\left(1-x_{1}\right)^{3} \ln \frac{m_{\tilde{g}}^{2}}{Q^{2}}+12 x_{1}^{2} \operatorname{Li}_{2}\left(1-x_{1}\right)+5 x_{1}^{3}-5 x_{1}^{2}+x_{1}-1+2\left(x_{1}^{3}+2 x_{1}^{2}\right) \ln x_{1}\right] \\
& -\left(x_{1} \longrightarrow x_{2}\right) \\
& \mathcal{R}_{2}=-\frac{C_{A}}{12\left(1-x_{1}\right)^{3}}\left[3\left(1-x_{1}^{2}+2 x_{1} \ln x_{1}\right)\left(2 \ln \frac{m_{t}^{2}}{m_{\tilde{g}}^{2}}-\mathcal{B}\left(\tau_{t}\right)-\frac{1}{2} \mathcal{K}^{1 \ell}\left(\tau_{t}\right)+2\right)\right. \\
& \left.+24 x_{1} \operatorname{Li}_{2}\left(1-x_{1}\right)+1-x_{1}^{2}+2 x_{1}\left(3 x_{1}+10\right) \ln x_{1}\right] \\
& +\frac{C_{F}}{18 x_{1}\left(1-x_{1}\right)^{4}}\left\{3 x_{1}\left[\left(1-x_{1}\right)\left(5 x_{1}-x_{1}^{2}+2\right)+6 x_{1} \ln x_{1}\right] \times\right. \\
& \times\left(2 \ln \frac{m_{t}^{2}}{m_{\tilde{g}}^{2}}-\mathcal{B}\left(\tau_{t}\right)-\frac{1}{2} \mathcal{G}_{1 / 2}^{1 \ell}\left(\tau_{t}\right)-\frac{1}{2} \mathcal{K}^{1 \ell}\left(\tau_{t}\right)+2\right)+6\left(1-x_{1}\right)^{4} \ln \frac{m_{\tilde{g}}^{2}}{Q^{2}} \\
& \left.+72 x_{1}^{2} \operatorname{Li}_{2}\left(1-x_{1}\right)-x_{1}\left(1-x_{1}\right)^{2}\left(11 x_{1}-26\right)-6\left(1-x_{1}\right)+6 x_{1}^{2}\left(2 x_{1}+9\right) \ln x_{1}\right\} \\
& +\left(x_{1} \longrightarrow x_{2}\right) .
\end{aligned}
$$

We recall that the function $\mathcal{G}_{1 / 2}^{1 \ell}(\tau)$ is defined in eq. (11), while $\mathcal{B}(\tau)$ and $\mathcal{K}^{1 \ell}(\tau)$ are defined as

$$
\mathcal{B}(\tau)=2+\sqrt{1-\tau} \ln \left(\frac{\sqrt{1-\tau}-1}{\sqrt{1-\tau}+1}\right), \quad \mathcal{K}^{1 \ell}(\tau)=\frac{\tau}{2} \ln ^{2}\left(\frac{\sqrt{1-\tau}-1}{\sqrt{1-\tau}+1}\right) .
$$

Finally, $\mathcal{F}_{1 / 2}^{(2 \ell, b)}$ can be expressed directly as a function of $\tau$ in terms of the other three functions:

$$
\mathcal{F}_{1 / 2}^{(2 \ell, b)}(\tau)=-\frac{3}{2}\left[2 \mathcal{G}_{1 / 2}^{1 \ell}(\tau)+\tau \mathcal{B}(\tau)-\mathcal{K}^{1 \ell}(\tau)\right]
$$


The top-stop-gluino contributions to the terms $Y_{\tilde{t}_{1}}$ and $Y_{c_{2 \theta_{t}}^{2}}$, arising from diagrams with a Higgsstop coupling (fig. 2b), read

$$
\begin{aligned}
& Y_{\tilde{t}_{1}}^{\tilde{g}}=\left(\frac{C_{F}}{4} \frac{s_{2 \theta_{t}}}{m_{t} m_{\tilde{g}}} \mathcal{G}_{1 / 2}^{1 \ell}\left(\tau_{t}\right)-\frac{2 C_{F}+C_{A}}{12 m_{\tilde{g}}^{2}}\right)\left(\frac{1}{1-x_{1}}+\frac{1}{\left(1-x_{1}\right)^{2}} \ln x_{1}\right) \\
& +\frac{C_{F}}{24 m_{\tilde{g}}^{2} x_{1}^{2}\left(1-x_{1}\right)^{3}}\left\{4\left(1-x_{1}\right)^{3}\left(1-\ln \frac{m_{\tilde{g}}^{2}}{Q^{2}}\right)-3 x_{1}^{2} \mathcal{G}_{1 / 2}^{1 \ell}\left(\tau_{t}\right)\left[\left(1-x_{1}\right)\left(3-x_{1}\right)+2 \ln x_{1}\right]\right\} \\
& +\frac{C_{F} s_{2 \theta_{t}} m_{t}}{6 m_{\tilde{g}}^{3} x_{1}^{2}\left(1-x_{1}\right)^{4}}\left\{3 x_{1}^{2}\left[\left(1-x_{1}\right)\left(x_{1}+5\right)+2\left(2 x_{1}+1\right) \ln x_{1}\right]\left(\frac{1}{4} \mathcal{G}_{1 / 2}^{1 \ell}\left(\tau_{t}\right)-\ln \frac{m_{t}^{2}}{m_{\tilde{g}}^{2}}\right)\right. \\
& +\left(1-x_{1}\right)^{4} \ln \frac{m_{\tilde{g}}^{2}}{Q^{2}}-12 x_{1}^{2}\left(2 x_{1}+1\right) \operatorname{Li}_{2}\left(1-x_{1}\right) \\
& \left.-\left(1-x_{1}\right)\left(14 x_{1}^{2}-3 x_{1}+1\right)-2 x_{1}^{2}\left(x_{1}^{2}+18 x_{1}+5\right) \ln x_{1}\right\} \\
& +\frac{C_{A} s_{2 \theta_{t}} m_{t}}{6 m_{\tilde{g}}^{3}\left(1-x_{1}\right)^{3}}\left\{3\left[2-2 x_{1}+\left(x_{1}+1\right) \ln x_{1}\right]\left(1+\ln \frac{m_{t}^{2}}{m_{\tilde{g}}^{2}}\right)\right. \\
& \left.+6\left(1+x_{1}\right) \operatorname{Li}_{2}\left(1-x_{1}\right)+2 x_{1}\left(1-x_{1}\right)+2\left(6 x_{1}+1\right) \ln x_{1}\right\} \\
& +\frac{C_{F} s_{2 \theta_{t}} m_{\phi}^{2} \mathcal{G}_{1 / 2}^{1 \ell}\left(\tau_{t}\right)}{48 m_{t} m_{\tilde{g}}^{3} x_{1}\left(1-x_{1}\right)^{4}}\left[\left(1-x_{1}\right)\left(x_{1}^{2}-5 x_{1}-2\right)-6 x_{1} \ln x_{1}\right], \\
& Y_{c_{2 \theta_{t}}^{2}}^{\tilde{g}}=-\frac{C_{F} m_{\tilde{g}}}{8 s_{2 \theta_{t}} m_{t}} \mathcal{G}_{1 / 2}^{1 \ell}\left(\tau_{t}\right)\left(\frac{x_{1}}{1-x_{1}} \ln x_{1}-\frac{x_{2}}{1-x_{2}} \ln x_{2}\right) \\
& +\left\{\frac { C _ { F } m _ { t } } { 1 2 s _ { 2 \theta _ { t } } m _ { \tilde { g } } x _ { 1 } ( 1 - x _ { 1 } ) ^ { 3 } } \left[3 x_{1}\left(x_{1}^{2}-2 x_{1} \ln x_{1}-1\right)\left(\frac{1}{4} \mathcal{G}_{1 / 2}^{1 \ell}\left(\tau_{t}\right)-\ln \frac{m_{t}^{2}}{m_{\tilde{g}}^{2}}\right)+\left(1-x_{1}\right)^{3} \ln \frac{m_{\tilde{g}}^{2}}{Q^{2}}\right.\right. \\
& \left.+12 x_{1}^{2} \operatorname{Li}_{2}\left(1-x_{1}\right)-\left(1-2 x_{1}\right)\left(1-x_{1}\right)^{2}+2 x_{1}^{2}\left(x_{1}+5\right) \ln x_{1}\right] \\
& +\frac{C_{A} m_{t} x_{1}}{12 s_{2 \theta_{t}} m_{\tilde{g}}\left(1-x_{1}\right)^{2}}\left[\left(x_{1}-1-\ln x_{1}\right)\left(3 \ln \frac{m_{t}^{2}}{m_{\tilde{g}}^{2}}+1\right)-6 \operatorname{Li}_{2}\left(1-x_{1}\right)-2\left(x_{1}+2\right) \ln x_{1}\right] \\
& +\frac{C_{F} m_{\phi}^{2} \mathcal{G}_{1 / 2}^{1 \ell}\left(\tau_{t}\right)}{32 s_{2 \theta_{t}} m_{\tilde{g}} m_{t}\left(1-x_{1}\right)^{2}}\left[\frac{\left(1-x_{1}\right)\left(x_{1}+x_{2}-2 x_{1} x_{2}\right)}{\left(1-x_{2}\right)\left(x_{1}-x_{2}\right)}+\frac{2 x_{1}\left(x_{1}^{2}+x_{1} x_{2}-2 x_{2}\right) \ln x_{1}}{\left(x_{1}-x_{2}\right)^{2}}\right] \\
& \left.-\left(x_{1} \longleftrightarrow x_{2}\right)\right\}
\end{aligned}
$$

while $Y_{\tilde{t}_{2}}^{\tilde{g}}$ can be obtained by performing the substitutions $x_{1} \rightarrow x_{2}$ and $s_{2 \theta_{t}} \rightarrow-s_{2 \theta_{t}}$ in eq. (28).

We remark that some care is required in order to properly include in the form factors $\mathcal{H}_{i}$ only terms up to $\mathcal{O}\left(m_{\phi}^{2} / M^{2}\right), \mathcal{O}\left(m_{Z}^{2} / M^{2}\right)$ and $\mathcal{O}\left(m_{t}^{2} / M^{2}\right)$. In particular, in the calculation of the function $F_{t}^{2 \ell}$, eq. (13), we must use the full formulae for $Y_{\tilde{t}_{1}}^{\tilde{g}}$ and $Y_{c_{2 \theta_{t}}^{2}}^{\tilde{g}}$ in eqs. (28) and (29), respectively. On the other hand, in the calculation of the functions $G_{t}^{2 \ell}, \widetilde{F}_{t}^{2 \ell}$ and $\widetilde{G}_{t}^{2 \ell}$, eqs. (14)-(16), we must retain 
only the terms in the first two lines of eq. (28) for $Y_{\tilde{t}_{1}}^{\tilde{g}}$, and only the term in the first line of eq. (29) for $Y_{c_{2 \theta_{t}}^{2}}^{\tilde{g}}$.

As a first check of the correctness of our calculation, we verified that by taking the VHML (i.e., taking $\left.m_{\phi} \rightarrow 0\right)$ in the formulae presented in this section, which implies $\mathcal{G}_{1 / 2}^{1 \ell}\left(\tau_{t}\right) \rightarrow-4 / 3, \mathcal{K}^{1 \ell}\left(\tau_{t}\right) \rightarrow$ $-2, \mathcal{B}\left(\tau_{t}\right) \rightarrow 0$ and $\mathcal{F}_{1 / 2}^{(2 \ell, b)} \rightarrow 0$, we obtain for the top-stop-gluino contributions the same result that we would obtain by expanding the VHML results of ref. [21] in powers of $m_{t}$ up to and including $\mathcal{O}\left(m_{t}^{2} / M^{2}\right)$. It is also straightforward to check that, by performing the trivial replacement $t \rightarrow b$ in the formulae presented in this section and then dropping all terms that contribute to the form factors beyond $\mathcal{O}\left(m_{b}^{2} / m_{\phi}^{2}\right), \mathcal{O}\left(m_{b} / M\right)$ and $\mathcal{O}\left(m_{z}^{2} / M^{2}\right)$, we recover the results of ref. [27] for the bottomsbottom-gluino contributions. To this effect it must be kept in mind that $\mathcal{G}_{1 / 2}^{1 \ell}\left(\tau_{b}\right)$ and $\mathcal{K}^{1 \ell}\left(\tau_{b}\right)$ are of $\mathcal{O}\left(m_{b}^{2} / m_{\phi}^{2}\right)$, while $\mathcal{B}\left(\tau_{b}\right)=2-\ln \left(-m_{\phi}^{2} / m_{b}^{2}\right)+\mathcal{O}\left(m_{b}^{2} / m_{\phi}^{2}\right)$

\subsection{On-shell renormalization scheme for the stop parameters}

If the parameters entering the one-loop part of the form factors are expressed in a renormalization scheme different from $\overline{\mathrm{DR}}$, the two-loop results presented in the previous section must be shifted in a way analogous to that described in section 3.2 of ref. [21], to which we point the reader for details. Indeed, in our "on-shell" (OS) scheme we adopt the same prescriptions as in ref. [21] for the input parameters that are subject to $\mathcal{O}\left(\alpha_{s}\right)$ corrections: the top and stop masses are defined as the poles of the corresponding propagators; the counterterm of the stop mixing angle $\theta_{t}$ is chosen as to cancel the anti-hermitian part of the stop wave-function renormalization matrix; the trilinear coupling $A_{t}$ is treated as a derived quantity, related to the other parameters in the top/stop sector by

$$
s_{2 \theta_{t}}=\frac{2 m_{t}\left(A_{t}+\mu \cot \beta\right)}{m_{\tilde{t}_{1}}^{2}-m_{\tilde{t}_{2}}^{2}} .
$$

Some differences with respect to the treatment in ref. [21] arise, however, due to the fact that the results presented in that paper were obtained in the VHML for arbitrary values of the top mass, while the results presented here are valid up to and including terms of $\mathcal{O}\left(m_{\phi}^{2} / M^{2}\right)$ and $\mathcal{O}\left(m_{t}^{2} / M^{2}\right)$, without assuming a hierarchy between $m_{\phi}$ and $m_{t}$. Defining the $\overline{\mathrm{DR}}-\mathrm{OS}$ shift for a generic parameter $x$ according to $x^{\overline{\mathrm{DR}}}=x^{\mathrm{OS}}+\left(\alpha_{s} / \pi\right) \delta x$, we need here to expand the various shifts in powers of $m_{t}$. Up to the order relevant to our calculation, the explicit expressions for the shifts in the stop masses and mixing angle read

$$
\begin{aligned}
\delta m_{\tilde{t}_{1}}^{2}=\frac{C_{F}}{4} m_{\tilde{t}_{1}}^{2}\{ & 3 \ln \frac{m_{\tilde{t}_{1}}^{2}}{Q^{2}}-3-c_{2 \theta_{t}}^{2}\left(\ln \frac{m_{\tilde{t}_{1}}^{2}}{Q^{2}}-1\right)-s_{2 \theta_{t}}^{2} \frac{m_{\tilde{t}_{2}}^{2}}{m_{\tilde{t}_{1}}^{2}}\left(\ln \frac{m_{\tilde{t}_{2}}^{2}}{Q^{2}}-1\right) \\
& -6 \frac{m_{\tilde{g}}^{2}}{m_{\tilde{t}_{1}}^{2}}-2\left(1-2 \frac{m_{\tilde{g}}^{2}}{m_{\tilde{t}_{1}}^{2}}\right) \ln \frac{m_{\tilde{g}}^{2}}{Q^{2}}-2\left(1-\frac{m_{\tilde{g}}^{2}}{m_{\tilde{t}_{1}}^{2}}\right)^{2} \ln \left|1-\frac{m_{\tilde{t}_{1}}^{2}}{m_{\tilde{g}}^{2}}\right| \\
& \left.-\frac{4 s_{2 \theta_{t}} m_{t} m_{\tilde{g}}}{m_{\tilde{t}_{1}}^{2}}\left[\ln \frac{m_{\tilde{g}}^{2}}{Q^{2}}+\left(1-\frac{m_{\tilde{g}}^{2}}{m_{\tilde{t}_{1}}^{2}}\right) \ln \left|1-\frac{m_{\tilde{t}_{1}}^{2}}{m_{\tilde{g}}^{2}}\right|-2\right]\right\}
\end{aligned}
$$




$$
\begin{aligned}
\delta \theta_{t} & =\frac{C_{F}}{4} \frac{c_{2 \theta_{t}} s_{2 \theta_{t}}}{\left(m_{\tilde{t}_{1}}^{2}-m_{\tilde{t}_{2}}^{2}\right)}\left\{m_{\tilde{t}_{1}}^{2}\left(\ln \frac{m_{\tilde{t}_{1}}^{2}}{Q^{2}}-1\right)-\frac{2 m_{t} m_{\tilde{g}}}{s_{2 \theta_{t}}}\left[\ln \frac{m_{\tilde{g}}^{2}}{Q^{2}}+\left(1-\frac{m_{\tilde{g}}^{2}}{m_{\tilde{t}_{1}}^{2}}\right) \ln \left|1-\frac{m_{\tilde{t}_{1}}^{2}}{m_{\tilde{g}}^{2}}\right|-2\right]\right\} \\
& +\left(\tilde{t}_{1} \longleftrightarrow \tilde{t}_{2}, \quad s_{2 \theta_{t}} \longrightarrow-s_{2 \theta_{t}}, \quad c_{2 \theta_{t}} \longrightarrow-c_{2 \theta_{t}}\right)
\end{aligned}
$$

where the analogous expression for $\delta m_{\tilde{t}_{2}}^{2}$ can be obtained by performing the substitutions $\tilde{t}_{1} \leftrightarrow \tilde{t}_{2}$ and $s_{2 \theta_{t}} \rightarrow-s_{2 \theta_{t}}$ in eq. (311). We also define $\delta s_{2 \theta_{t}}=2 c_{2 \theta_{t}} \delta \theta_{t}$ and $\delta c_{2 \theta_{t}}=-2 s_{2 \theta_{t}} \delta \theta_{t}$. The shift for the top mass reads

$$
\delta m_{t}=\frac{C_{F}}{4} m_{t}\left(3 \ln \frac{m_{t}^{2}}{Q^{2}}-5\right)+\delta m_{t}^{S U S Y},
$$

where the SUSY contribution $\delta m_{t}^{S U S Y}$ was given in eq. (23). Finally, the shift for the trilinear coupling $A_{t}$ can be expressed in terms of the other shifts according to

$$
\delta A_{t}=\left(\frac{\delta m_{\tilde{t}_{1}}^{2}-\delta m_{\tilde{t}_{2}}^{2}}{m_{\tilde{t}_{1}}^{2}-m_{\tilde{t}_{2}}^{2}}+\frac{\delta s_{2 \theta_{t}}}{s_{2 \theta_{t}}}-\frac{\delta m_{t}}{m_{t}}\right)\left(A_{t}+\mu \cot \beta\right) .
$$

If the one-loop form factors are evaluated in terms of OS parameters, the two-loop functions in eqs. (13)-(16) must be replaced by

$$
\begin{aligned}
& F_{t}^{2 \ell} \longrightarrow F_{t}^{2 \ell}+\frac{1}{6}\left[\frac{\delta m_{\tilde{t}_{1}}^{2}}{m_{\tilde{t}_{1}}^{4}}-\frac{\delta m_{\tilde{t}_{2}}^{2}}{m_{\tilde{t}_{2}}^{4}}-\left(\frac{\delta m_{t}}{m_{t}}+\frac{\delta s_{2 \theta_{t}}}{s_{2 \theta_{t}}}\right)\left(\frac{1}{m_{\tilde{t}_{1}}^{2}}-\frac{1}{m_{\tilde{t}_{2}}^{2}}\right)-\frac{2 m_{\phi}^{2}}{15} \frac{\delta m_{t}}{m_{t}}\left(\frac{1}{m_{\tilde{t}_{1}}^{4}}-\frac{1}{m_{\tilde{t}_{2}}^{4}}\right)\right], \\
& G_{t}^{2 \ell} \longrightarrow G_{t}^{2 \ell}+\frac{1}{6}\left[\frac{\delta m_{\tilde{t}_{1}}^{2}}{m_{\tilde{t}_{1}}^{4}}+\frac{\delta m_{\tilde{t}_{2}}^{2}}{m_{\tilde{t}_{2}}^{4}}-2 \frac{\delta m_{t}}{m_{t}}\left(\frac{1}{m_{\tilde{t}_{1}}^{2}}+\frac{1}{m_{\tilde{t}_{2}}^{2}}\right)\right]-\frac{2}{3} \mathcal{F}_{1 / 2}^{(2 \ell, b)}\left(\tau_{t}\right) \frac{\delta m_{t}}{m_{t}^{3}}, \\
& \widetilde{F}_{t}^{2 \ell} \longrightarrow \widetilde{F}_{t}^{2 \ell}+\frac{1}{6}\left[\frac{\delta m_{\tilde{t}_{1}}^{2}}{m_{\tilde{t}_{1}}^{4}}-\frac{\delta m_{\tilde{t}_{2}}^{2}}{m_{\tilde{t}_{2}}^{4}}-\frac{\delta c_{2 \theta_{t}}}{c_{2 \theta_{t}}}\left(\frac{1}{m_{\tilde{t}_{1}}^{2}}-\frac{1}{m_{\tilde{t}_{2}}^{2}}\right)\right], \\
& \widetilde{G}_{t}^{2 \ell} \longrightarrow \widetilde{G}_{t}^{2 \ell}+\frac{1}{6}\left[\frac{\delta m_{\tilde{t}_{1}}^{2}}{m_{\tilde{t}_{1}}^{4}}+\frac{\delta m_{\tilde{t}_{2}}^{2}}{m_{\tilde{t}_{2}}^{4}}\right] .
\end{aligned}
$$

In addition, the two-loop form factor $\mathcal{H}_{2}^{2 \ell}$ gets contributions originating from the shift in $A_{t}$ :

$$
\mathcal{H}_{2}^{2 \ell} \longrightarrow \mathcal{H}_{2}^{2 \ell}-\frac{m_{t} s_{2 \theta_{t}}}{6 s_{\beta}}\left[\delta A_{t}\left(\frac{1}{m_{\tilde{t}_{1}}^{2}}-\frac{1}{m_{\tilde{t}_{2}}^{2}}\right)+\frac{2 m_{\phi}^{2}}{15} \delta A_{t}\left(\frac{1}{m_{\tilde{t}_{1}}^{4}}-\frac{1}{m_{\tilde{t}_{2}}^{4}}\right)\right]
$$

Once again, care is required in order to properly include in the form factors $\mathcal{H}_{i}$ only terms up to $\mathcal{O}\left(m_{\phi}^{2} / M^{2}\right), \mathcal{O}\left(m_{Z}^{2} / M^{2}\right)$ and $\mathcal{O}\left(m_{t}^{2} / M^{2}\right)$. In particular:

i) the shifts $\delta m_{\tilde{t}_{1}}^{2}, \delta m_{\tilde{t}_{2}}^{2}$ and $\delta \theta_{t}$ must be computed up to $\mathcal{O}\left(m_{t}\right)$ in $F_{t}^{2 \ell}$, eq. (35), while they must be truncated at order zero in $m_{t}$ in $G_{t}^{2 \ell}, \widetilde{F}_{t}^{2 \ell}$ and $\widetilde{G}_{t}^{2 \ell}$, eqs. (36)-(38);

ii) in $F_{t}^{2 \ell}$, eq. (35), the first occurrence of $\delta m_{t}$ must be computed up to $\mathcal{O}\left(m_{t}^{2}\right)$, while the second occurrence, in the term proportional to $m_{\phi}^{2}$, must be truncated at order zero in $m_{t}$; 
iii) in $G_{t}^{2 \ell}$, eq. (36), the first occurrence of $\delta m_{t}$ must be truncated at $\mathcal{O}\left(m_{t}\right)$, while the second occurrence, in the term proportional to $\mathcal{F}_{1 / 2}^{(2 \ell, b)}\left(\tau_{t}\right)$, must be computed up to $\mathcal{O}\left(m_{t}^{3}\right)$;

iv) finally, in eq. (39) the first occurrence of $\delta A_{t}$ must be computed up to $\mathcal{O}\left(m_{t}\right)$ by means of eq. (34), while the second occurrence, in the term proportional to $m_{\phi}^{2}$, must be truncated at $\mathcal{O}\left(m_{t}^{-1}\right)$.

Ref. [28] provides formulae for the two-loop SUSY contributions to the form factors for scalar production in gluon fusion, in the OS renormalization scheme, also based on an asymptotic expansion in the superparticle masses but restricted to the limit of zero squark mixing and degenerate superparticle masses. We checked that our OS results agree with those of ref. [28] in the simplified limit considered in that paper, after taking into account a difference in the overall normalization factor and the fact that ref. [28] employs the opposite convention for the sign of $\mu$ with respect to our eq. (30).

\section{A numerical example}

We will now discuss a numerical evaluation of the two-loop SUSY contributions to the form factors for scalar Higgs production in a representative region of the MSSM parameter space.

The SM parameters entering our calculation include the $Z$ boson mass $m_{Z}=91.1876 \mathrm{GeV}$, the $W$ boson mass $m_{W}=80.399 \mathrm{GeV}$ and the strong coupling constant $\alpha_{s}\left(m_{Z}\right)=0.118$ [30]. For the pole mass of the top quark we take $M_{t}=173.2 \mathrm{GeV}$ [31]. For the relevant SUSY parameters we choose

$$
m_{Q}=m_{U}=\mu=1 \mathrm{TeV}, \quad A_{t}=2 \mathrm{TeV}, \quad m_{\tilde{g}}=800 \mathrm{GeV}, \quad \tan \beta=5,
$$

where $m_{Q}$ and $m_{U}$ are the soft SUSY-breaking masses for the left and right stops, respectively. For a given value of the pseudoscalar mass $m_{A}$, the scalar masses $m_{h}$ and $m_{H}$ and the mixing angle $\alpha$ are computed including the leading one-loop corrections of $\mathcal{O}\left(\alpha_{t}\right)$ and the leading two-loop corrections of $\mathcal{O}\left(\alpha_{s} \alpha_{t}\right)$ [32].

In fig. 3 we show the real part of the SUSY (i.e., all except top-gluon) contributions to the two-loop form factor for heaviest-Higgs production, $\mathcal{H}_{H}^{2 \ell}$, as a function of $m_{H}$. Since, as mentioned above, $m_{H}$ is not a free parameter in our calculation, its variation is obtained by varying $m_{A}$ between $100 \mathrm{GeV}$ and $500 \mathrm{GeV}$. For simplicity, in the computation of the form factor we neglected the small D-terminduced electroweak contributions. The left plot in figure 3 is obtained assuming that the parameters $m_{t}, m_{\tilde{t}_{1}}, m_{\tilde{t}_{2}}$ and $\theta_{t}$ entering the one-loop part of the form factor, $\mathcal{H}_{H}^{1 \ell}$, are expressed in the $\overline{\mathrm{DR}}$ renormalization scheme at the scale $Q=1 \mathrm{TeV}$. In this case we extract the $\overline{\mathrm{DR}}$ top mass $m_{t}(Q)$ from the input value for the pole mass $M_{t}$ by means of eq. (B2) of ref. [32], and we interpret the input parameters $m_{Q}, m_{U}$ and $A_{t}$ in eq. (40) directly as running parameters evaluated at the scale $Q$. The right plot, on the other hand, is obtained assuming that the parameters $m_{t}, m_{\tilde{t}_{1}}, m_{\tilde{t}_{2}}$ and $\theta_{t}$ entering $\mathcal{H}_{H}^{1 \ell}$ are expressed in the OS scheme described in section 3.2 of ref. [21]. In this case we identify $m_{t}$ directly with the pole mass $M_{t}$, and we interpret the input parameters $m_{Q}, m_{U}$ and $A_{t}$ in eq. (40) as the parameters that can be obtained by rotating the diagonal matrix of the physical stop masses by the "physical" angle $\theta_{t}$, defined through eq. (37) of ref. [21]. 

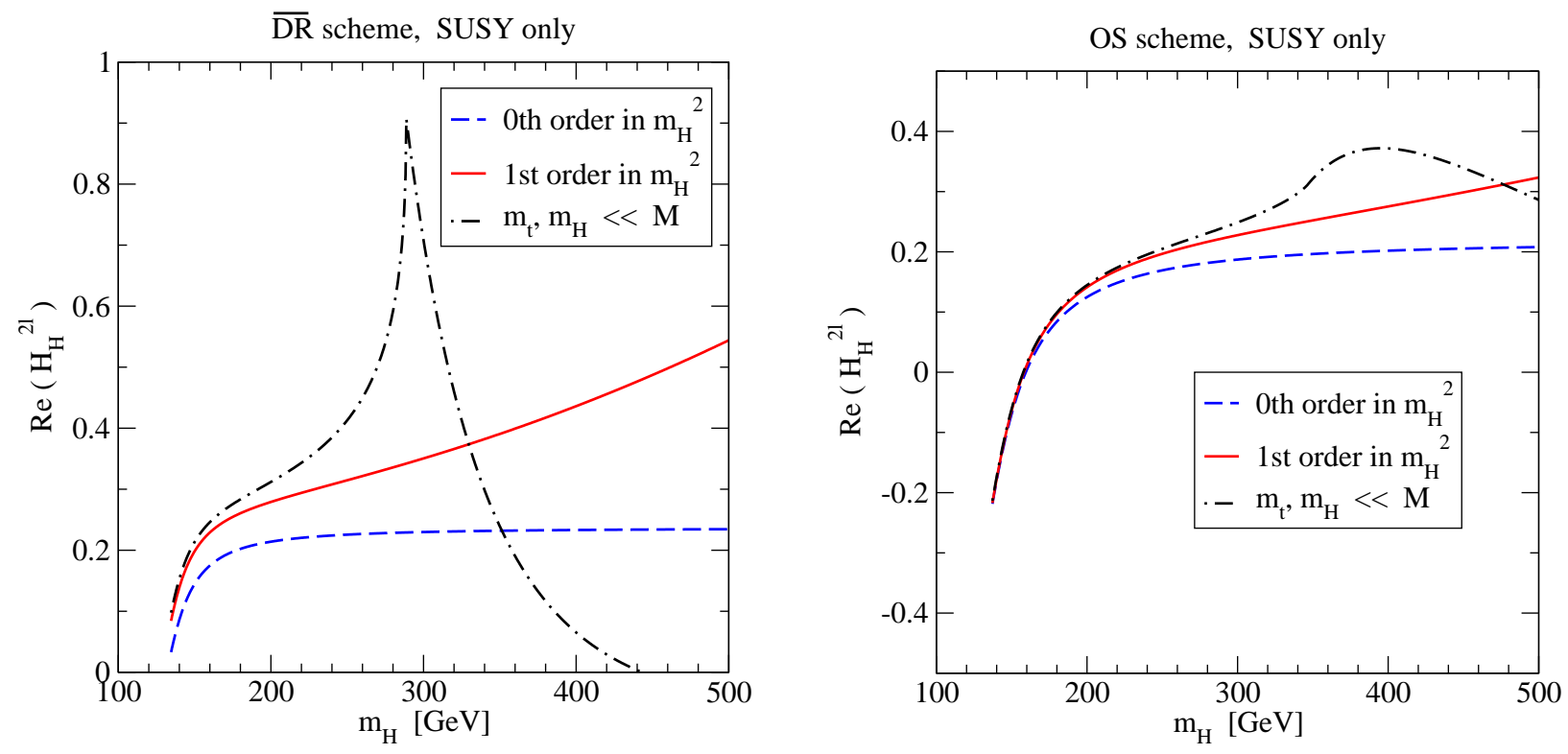

Figure 3: Real part of the SUSY contributions to $\mathcal{H}_{H}^{2 \ell}$, plotted as a function of $m_{H}$. The choice of SUSY parameters and the meaning of the different curves are explained in the text. The plot on the left refers to the $\overline{\mathrm{DR}}$ scheme, while the plot on the right refers to the OS scheme.

In each plot, the dashed (blue) line represents the result obtained in the VHML, as given in ref. [21], while the solid (red) line represents the result computed at the first order of a Taylor expansion in $m_{H}^{2}$, i.e. it includes the effect of terms of $\mathcal{O}\left(m_{H}^{2} / m_{t}^{2}\right)$ and $\mathcal{O}\left(m_{H}^{2} / M^{2}\right)$ which were also computed in ref. [21] but proved too lengthy to be presented in analytic form. The dot-dashed (black) line represents instead the result of the asymptotic expansion in the superparticle masses derived in this paper. The latter is applicable when both $m_{t}$ and $m_{H}$ are smaller than the generic superparticle mass $M$, as is indeed the case here since $M \approx 1 \mathrm{TeV}$, but it does not require any specific hierarchy between $m_{H}$ and $m_{t}$.

The comparison between the dashed and solid lines shows that, as $m_{H}$ increases, the effect of the terms of $\mathcal{O}\left(m_{H}^{2} / m_{t}^{2}\right)$ and $\mathcal{O}\left(m_{H}^{2} / M^{2}\right)$ becomes more and more relevant, and the VHML does not provide an accurate approximation to $\mathcal{H}_{H}^{2 \ell}$. Furthermore, the comparison between the dot-dashed and solid lines shows that, even if the inclusion of the first-order terms pushes the validity of the Taylor expansion up to larger values of $m_{H}$, the Taylor expansion fails anyway when $m_{H}$ gets close to the threshold for the production of a real top-quark pair in the loops. In that case one can use the result of our asymptotic expansion in $M$, provided that the latter is still considerably larger than $m_{H}$.

A few additional comments are in order concerning the comparison between the left $(\overline{\mathrm{DR}})$ and right (OS) plots in fig. 3. There is no reason to expect the plots to look similar to each other, first of all because the difference between the values of $\mathcal{H}_{H}^{2 \ell}$ in the two schemes is compensated for, up to higherorder terms, by a shift in the value of the one-loop form factor, $\mathcal{H}_{H}^{1 \ell}$, and also because the different interpretation of the input parameters in the two schemes means that, by using the numerical inputs in 
eq. (40) for both schemes, we are in fact considering two different points of the MSSM parameter space. This said, a striking difference between the two schemes is visible in the behavior of the asymptotic expansion (i.e., the dot-dashed line) around the threshold for the production of a real top-quark pair in the loops. The fact that in the $\overline{\mathrm{DR}}$ plot the threshold is located at a lower value of $m_{H}$ than in the OS plot is an artifact, due to lower value of the MSSM running top mass with respect to the pole top mass (indeed, for our choice of parameters $m_{t}(Q)=144.3 \mathrm{GeV}$ ). The much sharper behavior around the threshold of the dot-dashed line in the $\overline{\mathrm{DR}}$ plot, on the other hand, can be traced back to the contribution of the first term in the right-hand side of eq. (22) for $Y_{t}^{\tilde{g}}$. That term reflects the fact that the running top mass of the MSSM (i.e., including the stop-gluino contribution) is used in the top-quark contribution to $\mathcal{H}_{H}^{1 \ell}$, and it is canceled out by the last term of eq. (36) if the pole top mass (or, for that matter, the running top mass of the SM) is used instead. Indeed, we checked that, in a "mixed" renormalization scheme in which the stop contributions to $\mathcal{H}_{H}^{1 \ell}$ are expressed in term of running parameters (including the MSSM running top mass) but the top-quark contribution is expressed in terms of the pole top mass, the qualitative behavior of the dot-dashed line around the threshold would be similar to the one in the OS plot.

To conclude this discussion, we show in fig. 4 the real part of the SUSY contributions to the twoloop form factor for lightest-Higgs production, $\mathcal{H}_{h}^{2 \ell}$, as a function of the pseudoscalar mass $m_{A}$, which is varied in the same range used to produce fig. 3. The meaning of the different curves is the same as in fig. 3, and again the left plot is obtained assuming that the parameters entering the one-loop form factor $\mathcal{H}_{h}^{1 \ell}$ are expressed in the $\overline{\mathrm{DR}}$ scheme, while the right plot is obtained assuming that they are expressed in the OS scheme.

In the MSSM the mass of the lightest Higgs scalar $h$ is bounded from above, and for large enough values of the pseudoscalar mass it becomes independent of $m_{A}$, as do the couplings of $h$ to the top quark and to the stops. Indeed, for the choice of SUSY parameters in eq. (40) our crude $\mathcal{O}\left(\alpha_{t}+\alpha_{t} \alpha_{s}\right)$ calculation of the Higgs mass yields $m_{h}<123.8 \mathrm{GeV}$ in the $\overline{\mathrm{DR}}$ plot and $m_{h}<122.5 \mathrm{GeV}$ in the OS plot, and all the curves in fig. 4 become essentially flat for $m_{A}>250 \mathrm{GeV}$. Due to the relative smallness of $m_{h}$ no real-particle threshold is crossed, thus the result of the asymptotic expansion (dot-dashed line) is rather close to the result of the Taylor expansion at the first order in $m_{h}^{2}$ (solid line).

However, a comparison between the left and right plots of fig. 4 shows that in the $\overline{\mathrm{DR}}$ calculation the VHML result (dashed line) provides a less-than-perfect approximation to $\mathcal{H}_{h}^{2 \ell}$, while in the OS calculation the effect of the terms proportional to $m_{h}^{2}$ is small, and the VHML result essentially overlaps with the other two results. This difference between the two schemes can again be traced to the contribution of the first term in the right-hand side of eq. (22), i.e. to the choice of renormalization scheme for the top mass entering the top-quark contribution to $\mathcal{H}_{h}^{1 \ell}$. Even in this case we checked that, in a "mixed" scheme in which the top-quark contribution to $\mathcal{H}_{h}^{1 \ell}$ is expressed in terms of the pole top mass while the stop contributions are expressed in terms of running parameters, the VHML would provide as good an approximation to $\mathcal{H}_{h}^{2 \ell}$ as it does in the full OS scheme. 

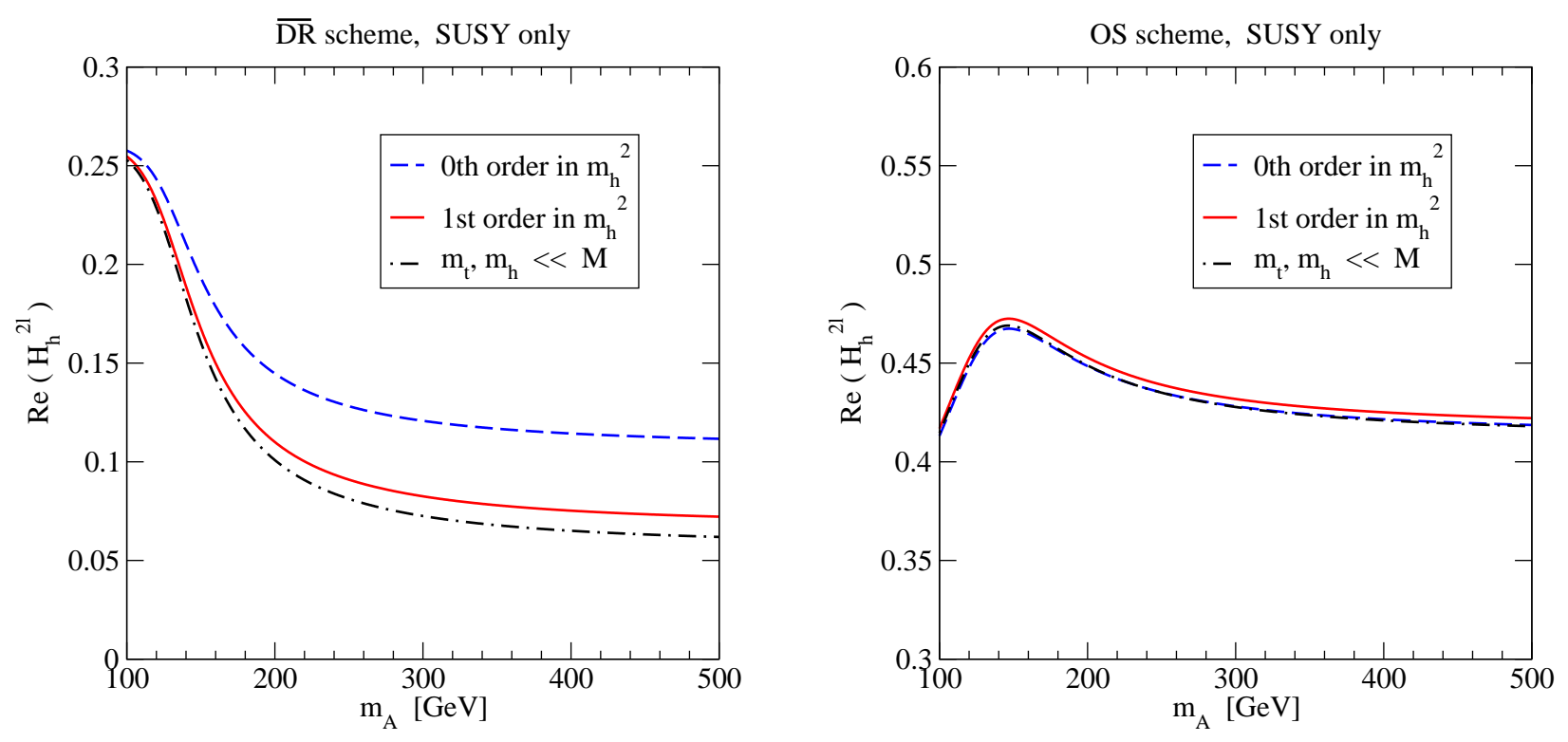

Figure 4: Real part of the SUSY contributions to $\mathcal{H}_{h}^{2 \ell}$, plotted as a function of $m_{A}$. The choice of SUSY parameters and the meaning of the different curves are explained in the text. The plot on the left refers to the $\overline{\mathrm{DR}}$ scheme, while the plot on the right refers to the OS scheme.

\section{Conclusions}

The calculation of the production cross section for the MSSM Higgs bosons is not quite as advanced as in the SM. Indeed, a full computation of the two-loop quark-squark-gluino contributions, valid for arbitrary values of all the relevant particle masses, has not been made publicly available so far. Moreover, the complexity of such a computation is going to be reflected in results that will probably be too lengthy and computer-time-consuming to be efficiently implemented in event generators. An alternative approach consists in deriving approximate analytic results that can be easily implemented in computer codes, and that are valid in specific regions of the MSSM parameter space such as, e.g., when the Higgs bosons are somewhat lighter than the squarks and the gluino.

In this paper we presented a new calculation of the two-loop top-stop-gluino contributions to the form factors for Higgs scalar production in gluon fusion. We exploited techniques developed in our earlier computations of the production cross section for the MSSM Higgs bosons [27, 22] to obtain explicit and compact analytic results based on an asymptotic expansion in the heavy particle masses, up to and including terms of $\mathcal{O}\left(m_{\phi}^{2} / M^{2}\right), \mathcal{O}\left(m_{z}^{2} / M^{2}\right)$ and $\mathcal{O}\left(m_{t}^{2} / M^{2}\right)$. We compared our new results with the VHML results of ref. [21, as well as with the results of a Taylor expansion in the Higgs mass, up to and including terms of $\mathcal{O}\left(m_{\phi}^{2} / m_{t}^{2}\right)$ and $\mathcal{O}\left(m_{\phi}^{2} / M^{2}\right)$, and we discussed the regions of applicability of the different expansions. We also discussed the effect of choosing different renormalization schemes for the parameters in the top/stop sector. 
From the example presented in section 4 it appears that, in the case of the heaviest Higgs boson $H$, the use of our asymptotic expansion becomes mandatory when $m_{H}$ approaches (or crosses) the threshold for the production of a real top-quark pair in the loops. It also appears that choosing the OS scheme for the parameters in the top/stop sector leads to a milder behavior of the two-loop form factor $\mathcal{H}_{H}^{2 \ell}$ around the threshold. In the case of the lightest Higgs boson $h$, whose mass is bounded from above in the MSSM, we need not worry about thresholds. However, our discussion showed that in the $\overline{\mathrm{DR}}$ scheme the VHML provides a worse approximation to $\mathcal{H}_{h}^{2 \ell}$ than it does in the OS scheme.

Finally, we remark that the results derived in this paper for the production cross section can be straightforwardly adapted to the NLO computation of the gluonic and photonic decay widths of the MSSM Higgs bosons, in analogy to what described in section 5 of ref. [21].

\section{Acknowledgments}

This work was partially supported by the Research Executive Agency (REA) of the European Union under the Grant Agreement number PITN-GA-2010-264564 (LHCPhenoNet).

\section{References}

[1] S. Dittmaier et al. [LHC Higgs Cross Section Working Group], arXiv:1101.0593 [hep-ph], arXiv:1201.3084 [hep-ph].

[2] H. M. Georgi, S. L. Glashow, M. E. Machacek and D. V. Nanopoulos, Phys. Rev. Lett. 40 (1978) 692.

[3] S. Dawson, Nucl. Phys. B 359 (1991) 283; A. Djouadi, M. Spira and P. M. Zerwas, Phys. Lett. B 264 (1991) 440.

[4] M. Spira, A. Djouadi, D. Graudenz and P. M. Zerwas, Nucl. Phys. B 453 (1995) 17 arXiv:hep-ph/9504378.

[5] R. Harlander and P. Kant, JHEP 0512 (2005) 015 [arXiv:hep-ph/0509189].

[6] C. Anastasiou, S. Beerli, S. Bucherer, A. Daleo and Z. Kunszt, JHEP 0701 (2007) 082 arXiv:hep-ph/0611236.

[7] U. Aglietti, R. Bonciani, G. Degrassi and A. Vicini, JHEP 0701 (2007) 021 arXiv:hep-ph/0611266.

[8] R. Bonciani, G. Degrassi and A. Vicini, JHEP 0711 (2007) 095 [arXiv:0709.4227 [hep-ph]].

[9] R. V. Harlander, Phys. Lett. B 492 (2000) 74. arXiv:hep-ph/0007289]; S. Catani, D. de Florian and M. Grazzini, JHEP 0105 (2001) 025 arXiv:hep-ph/0102227; R. V. Harlander and 
W. B. Kilgore, Phys. Rev. D 64 (2001) 013015 arXiv:hep-ph/0102241, Phys. Rev. Lett. 88 (2002) 201801 arXiv:hep-ph/0201206; C. Anastasiou and K. Melnikov, Nucl. Phys. B 646 (2002) 220 arXiv:hep-ph/0207004]; V. Ravindran, J. Smith and W. L. van Neerven, Nucl. Phys. B 665 (2003) 325 arXiv:hep-ph/0302135].

[10] S. Marzani, R. D. Ball, V. Del Duca, S. Forte and A. Vicini, Nucl. Phys. B 800 (2008) 127 arXiv:0801.2544 [hep-ph]], Nucl. Phys. Proc. Suppl. 186 (2009) 98 [arXiv:0809.4934 [hep-ph]]; R. V. Harlander and K. J. Ozeren, Phys. Lett. B 679 (2009) 467 [arXiv:0907.2997 [hep-ph]], JHEP 0911 (2009) 088 arXiv:0909.3420 [hep-ph]]; A. Pak, M. Rogal and M. Steinhauser, Phys. Lett. B 679 (2009) 473 [arXiv:0907.2998 [hep-ph]], JHEP 1002 (2010) 025 arXiv:0911.4662 [hepph]]; R. V. Harlander, H. Mantler, S. Marzani and K. J. Ozeren, Eur. Phys. J. C 66 (2010) 359 arXiv:0912.2104 [hep-ph]].

[11] M. Kramer, E. Laenen and M. Spira, Nucl. Phys. B 511, 523 (1998) arXiv:hep-ph/9611272; S. Catani, D. de Florian, M. Grazzini and P. Nason, JHEP 0307 (2003) 028 arXiv:hep-ph/0306211.

[12] A. Djouadi and P. Gambino, Phys. Rev. Lett. 73 (1994) 2528 arXiv:hep-ph/9406432; A. Djouadi, P. Gambino and B. A. Kniehl, Nucl. Phys. B 523 (1998) 17 arXiv:hep-ph/9712330.

[13] U. Aglietti, R. Bonciani, G. Degrassi and A. Vicini, Phys. Lett. B 595 (2004) 432 arXiv:hep-ph/0404071, Phys. Lett. B 600 (2004) 57 arXiv:hep-ph/0407162, arXiv:hep-ph/0610033; G. Degrassi and F. Maltoni, Phys. Lett. B 600 (2004) 255 arXiv:hep-ph/0407249; R. Bonciani, G. Degrassi, A. Vicini, Comput. Phys. Commun. 182 (2011) 1253 [arXiv:1007.1891 [hep-ph]].

[14] S. Actis, G. Passarino, C. Sturm and S. Uccirati, Phys. Lett. B 670 (2008) 12 [arXiv:0809.1301 [hep-ph]], Nucl. Phys. B 811 (2009) 182 [arXiv:0809.3667 [hep-ph]].

[15] S. Moch and A. Vogt, Phys. Lett. B 631 (2005) 48 arXiv:hep-ph/0508265; V. Ravindran, Nucl. Phys. B 752 (2006) 173 arXiv:hep-ph/0603041.

[16] C. Anastasiou, R. Boughezal and F. Petriello, JHEP 0904 (2009) 003 [arXiv:0811.3458 [hep-ph]].

[17] S. Dawson, A. Djouadi and M. Spira, Phys. Rev. Lett. 77 (1996) 16 arXiv:hep-ph/9603423.

[18] M. Muhlleitner and M. Spira, Nucl. Phys. B 790 (2008) 1 arXiv:hep-ph/0612254.

[19] R. V. Harlander and M. Steinhauser, Phys. Lett. B 574 (2003) 258 arXiv:hep-ph/0307346], Phys. Rev. D 68 (2003) 111701 arXiv:hep-ph/0308210, JHEP 0409 (2004) 066 arXiv:hep-ph/0409010.

[20] R. V. Harlander and F. Hofmann, JHEP 0603 (2006) 050 arXiv:hep-ph/0507041. 
[21] G. Degrassi and P. Slavich, Nucl. Phys. B 805 (2008) 267 [arXiv:0806.1495 [hep-ph]].

[22] G. Degrassi, S. Di Vita and P. Slavich, JHEP 1108 (2011) 128 [arXiv:1107.0914 [hep-ph]].

[23] A. Pak, M. Steinhauser and N. Zerf, Eur. Phys. J. C 71 (2011) 1602 [arXiv:1012.0639 [hep-ph]].

[24] C. Anastasiou, S. Beerli and A. Daleo, Phys. Rev. Lett. 100 (2008) 241806 arXiv:0803.3065 [hep-ph]].

[25] M. Muhlleitner, H. Rzehak and M. Spira, PoS RADCOR2009 (2010) 043 [arXiv:1001.3214 [hep-ph]].

[26] S. Chatrchyan et al. [CMS Collaboration], Phys. Rev. Lett. 107 (2011) 221804 [arXiv:1109.2352 [hep-ex]]; G. Aad et al. [Atlas Collaboration], JHEP 1111 (2011) 099 arXiv:1110.2299 [hep-ex]].

[27] G. Degrassi and P. Slavich, JHEP 1011 (2010) 044 arXiv:1007.3465 [hep-ph]].

[28] R. V. Harlander, F. Hofmann and H. Mantler, JHEP 1102 (2011) 055 [arXiv:1012.3361 [hep-ph]].

[29] E. Bagnaschi, G. Degrassi, P. Slavich and A. Vicini, JHEP 1202 (2012) 088 [arXiv:1111.2854 [hep-ph]].

[30] K. Nakamura et al. [Particle Data Group], J. Phys. G 37 (2010) 075021.

[31] [Tevatron Electroweak Working Group and for the CDF and D0 Collaborations], arXiv:1107.5255 [hep-ex].

[32] G. Degrassi, P. Slavich and F. Zwirner, Nucl. Phys. B 611 (2001) 403 arXiv:hep-ph/0105096. 